\title{
IDENTIFICACIÓN DE SEGMENTO DE MERCADO, EN LOS DISTRITOS DE EL TAMBO Y HUANCAYO, PARA PRODUCTOS NUTRACEÚTICOS*
}

\author{
Seguil Mirones, Carlos G. ${ }^{1}$ y Egas Peña, Enma²
}

Facultad de Industrias Alimentarias de la Universidad Nacional del Centro del Perú

\begin{abstract}
RESUMEN
El trabajo de investigación, determinó un segmento de mercado en los distritos metropolitanos de Huancayo y El Tambo de la provincia de Huancayo, el cual tiene interés en productos alimenticios con propiedades nutraceúticas. Este segmento fue determinado teniendo como descriptores: nivel educativo e ingreso familiar mensual. Características del segmento: Tamaño: $\mathbf{7 4} 6 \mathbf{6 5}$ habitantes que representa el 23,70\% del mercado total. Composición familiar: $\mathbf{5}$ integrantes por familia. Estilo de vida: conformado en su mayoría por un estilo de vida moderno. Lugares de compra: En forma proporcional en: bodegas, mercados de la zona y supermercados. Criterios para la decisión de compra: el criterio más importante es la calidad y que el producto sea natural, en segundo lugar se valora la marca del producto y finalmente el precio. Dolencias y enfermedades de interés del segmento de mercado: el mayor interés es por el cáncer, osteoporosis y colesterol. Ubicación: Se utilizó el plano de calles de la ciudad de Huancayo y distritos aledaños actualizado al 2005, las zonas son: 09 en Huancayo y 07 en El Tambo. Estrategia de comercialización: producto, alimentos que contienen principios activos. Precio: al inicio precio alto, luego se nivelará al precio de productos similares. Plaza: canal indirecto. Promoción publicidad, dirigida a los consumidores.
\end{abstract}

Palabras clave: investigación de mercado, segmentación de mercados, productos nutraceúticos.

\section{IDENTIFICATION OF MARKET NICHE, IN THE DISTRICTS OF EL TAMBO AND HUANCAYO, FOR NUTRACEUTICAL PRODUCTS}

\begin{abstract}
It has been determined; a niche market in the metropolitan districts of Huancayo and El Tambo in the province of Huancayo, which is interested in food products with nutraceutic properties, this segment was determined by taking as descriptors: educational attainment and monthly family income. Segment features: Size: 74645 inhabitants representing $\mathbf{2 3 , 7 0 \%}$ of the total market. Family Composition: $\mathbf{5}$ members per family. Lifestyle: mostly for a modern lifestyle. Purchasing places: proportionately: Wineries, markets and supermarkets in the area. Criteria for purchase decision: The most important criterion is the quality and nature of the product, second it is valued the brand and finally the price. Pains and disease of market segment: The greatest concern is for cancer, osteoporosis and cholesterol. Location: We used the Huancayo city street map and surrounding districts updated to 2005 , the areas are: 09 in Huancayo and 07 in El Tambo. Marketing strategy: Product; food containing bioactive components. Price; market skimming pricing. Market; indirect channel. Promotion; advertising aimed to consumers.
\end{abstract}

Key words: market research, segmentation of markets, nutraceutical products

\footnotetext{
* Este trabajo de investigación fue recibido el 20/12/2007 retornado para su revisión 26/10/2009 y aprobado para su publicación 16/11/2009

1Email: eseguil60@yahoo.com

2Email: epeña@uncp.edu.pe
} 


\section{INTRODUCCIÓN}

Actualmente todas las estrategias de comercialización, para el desarrollo de productos, como para la penetración de mercados, tiene como soporte una buena investigación de mercado, que permita definir si existe un segmento de mercado que valore los beneficios que ofrece el producto en dicho segmento, por lo tanto ahora ya no se piensa en servir un mercado amplio, sino pequeños mercados (segmentos o nichos), donde se pueda tener mayor probabilidad de éxito.

\section{MATERIAL Y MÉTODOS}

La población estuvo representada por la población total de los distritos metropolitanos de Huancayo y El Tambo; se utilizó una muestra probabilística tal como sugiere (1).

Los criterios base y descriptores, Según (2), se usó el criterio base: Beneficios buscados en los alimentos con características nutraceúticas, que corresponden al destino estratégico de la información, para el conocimiento general del mercado.

Los descriptores utilizados fueron:

- Nivel educativo (superior, secundario y primario).

- Ingreso familiar mensual (menos de S/. 1 000,00; igual o mayor a S/. 1 000,00 pero menos de S/. 2 000,00; igual o mayor S/. 2 000,00 pero menos de S/. 3 000,00 y más de S/. 3 000,00).

- Estilos de vida (afortunados, progresistas, modernos, adaptados, conservadores y modestos).

La determinación del tamaño muestral, se realizó siguiendo la metodología para la obtención de una muestra probabilística, la cual como dicen (3), las muestras probabilísticas tienen muchas ventajas, la principal es que puede medirse el tamaño de error en nuestras predicciones.

Por su parte (4), hace una distinción para la determinación del tamaño muestral, para universos infinitos, aquellos conformados por más de 100000 unidades.

Para el cálculo del tamaño muestral para la encuesta del estudio de mercado, se utilizó la siguiente fórmula:

$$
n=\frac{(1,96)^{2} \times p x q}{e^{2}}
$$

Donde:

$\mathrm{n}=$ tamaño muestral (habitantes del mercado en estudio) $1,96=$ para un coeficiente de confianza del 95,5\% ( \pm $1,96$ veces $\sigma)$

$p=0,50$ (probabilidad de ocurrencia, caso más desfavorable)

$q=0,50$ (probabilidad de no ocurrencia, caso más desfavorable)

pxq $=$ varianza de la muestra

$\mathrm{e}=0,04$ (error estándar)

Se obtuvo un tamaño muestral de $\mathbf{6 3 1}$ habitantes.

$$
N^{\circ} \text { de familias }=\frac{631 \text { habitantes }}{5,43 \text { habitantes } / \text { familia }}
$$

$\mathrm{N}^{\circ}$ de familias encuestadas $=116$

Se determinó las zonas para las encuestas, de acuerdo a (5), donde está dividido en cuadrículas asignados por la combinación de números y letras, teniendo cada cuadrícula un kilómetro cuadrado, en cada área se aplicó 4 a 5 encuestas, se tomó al azar los hogares a encuestar.

\section{RESULTADOS}

\section{Determinación del segmento}

El criterio para determinar el segmento se basó en dos descriptores, nivel educativo e ingresos familiares, en los cuales las intenciones de compra ha sido el 100\%.

Tamaño del segmento.- El tamaño total del segmento es: $\mathbf{7 4} \mathbf{6 4 5}$ habitantes que representa el $\mathbf{2 3 , 7 0 \%}$ del mercado total, en Huancayo 27454 habitantes y en el distrito de El Tambo 47191 habitantes pertenecen al segmento. 
Composición familiar.- El segmento se compone de 5 integrantes por familia. Las edades promedios en la familia se puede apreciar en la siguiente Figura. Lo que representa redondeando 01 integrante tiene entre $0 \mathrm{a}$ 10 años; 01 integrante tiene entre 11 a 20 años, 02 integrantes tienen entre 21 a 45 años y 01 integrante tiene más de 45 años de edad.

\section{Q 0-10 años प 11- 20 años $\square 21$ a 45 años $\square$ más de 45 años}

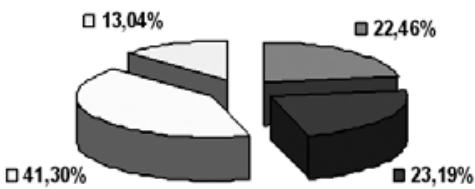

Gráfico 1. Composición de edades en el hogar del segmento de mercado.

Ingreso familiar.- El segmento tiene ingresos mayores a S/. 1000,00 que fue uno de los criterios de segmentación, la conformación según los diferentes niveles se muestra en la fiqura.

a Igual o + 3000 a Igual o + 2000 pero - 3000 q Igual o + 1000 pero -2000 a Menos de 1000

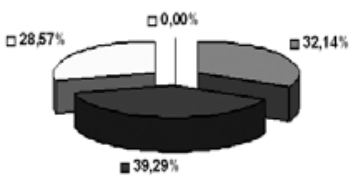

Gráfico 2. Composición de los ingresos de los integrantes del segmento de mercado

Nivel educativo.- Este fue otro criterio de segmentación, en la que solamente se tomó en cuenta los que tienen el nivel educativo superior y secundario, el segmento está conformado como se observa en la figura.

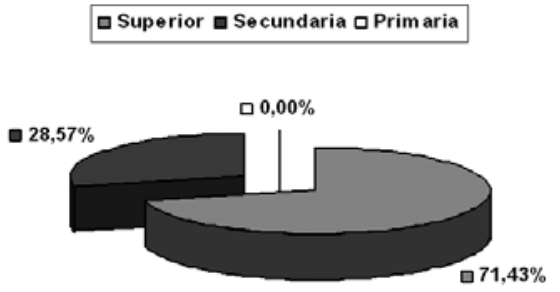

Gráfico 3. Nivel educativo del segmento de mercado.
Estilo de vida.- El segmento de mercado está conformado en su mayoría con un estilo de vida moderno, lo que permite posicionar nuevos productos, por su apertura a buscar productos novedosos.

\begin{tabular}{|c|c|}
\hline $\begin{array}{l}\text { a } a=\text { Progresistas } \\
\square d=\text { Afortunados }\end{array}$ & $\begin{array}{l}\square \mathrm{b}=\text { Modernas } \\
\square \mathrm{e}=\text { Adaptados }\end{array}$ \\
\hline
\end{tabular}

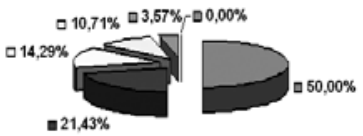

Gráfico 4. Estilos de vida del segmento de mercado

Lugares de compra.- Como se puede observar los lugares de compra de preferencia del segmento se da en forma proporcional en: Bodegas, mercados de la zona y supermercados.

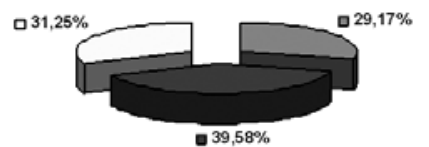

Gráfico 5. Lugares donde realizar sus compras el segmento de mercado.

Criterios para la decisión de compra.- El criterio más importante para tomar la decisión de compra está en la calidad y que el producto sea natural, en segundo lugar se valora la marca del producto y finalmente el precio.

$$
\square \text { Precio } \square \text { Calidad } \square \text { Marca }
$$



Gráfico 6. Criterios de decisión de compra del segmento de mercado. 
Dolencias y enfermedades de interés del segmento de mercado.- El segmento de mercado desea productos nutraceúticos para las dolencias y enfermedades que se muestra en la figura, cabe resaltar que el mayor interés es por el cáncer, osteoporosis y colesterol; sin embargo existen otras que bien podrían ser motivo de desarrollo de nuevos productos.

\begin{tabular}{|c|c|}
\hline $\begin{array}{l}\square \mathrm{a}=\text { Cáncer } \\
\square c=\text { Obesidad } \\
\square \mathrm{e}=\text { Triglicéridos } \\
\square \mathrm{g}=\text { Estreñimiento } \\
\square \mathrm{i}=\text { Dolencias estom acales } \\
\square \mathrm{k}=\text { Ácido úrico } \\
\square \mathrm{m}=\text { Depresión }\end{array}$ & $\begin{array}{l}\square b=\text { Diabetes } \\
\square d=\text { Colesterol } \\
\square f=\text { Osteoporosis } \\
\square h=\text { Dolencias hepáticas } \\
\square j=\text { Cálculos biliares } \\
\square l=\text { Problem as cardiovas culares } \\
\square n=\text { otros }\end{array}$ \\
\hline
\end{tabular}

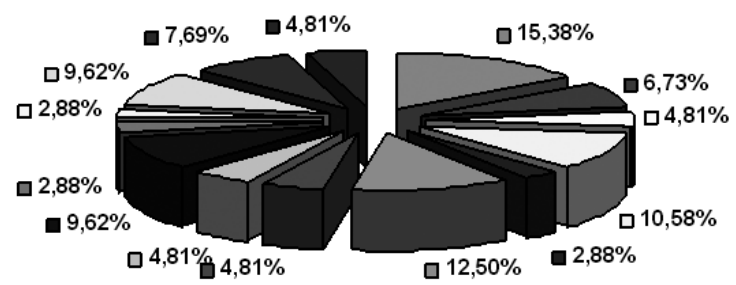

Gráfico 6. Interés en dolencias y enfermedades del segmento de mercado.

Ubicación del segmento.- Para esta ubicación se utilizó el plano de calles de la ciudad de Huancayo y distritos aledaños actualizado al 2005, se tiene que indicar que no existe zonas bien definidas del segmento, sino que se encuentran bastante diseminados.

\section{Estrategia de comercialización}

La estrategia de comercialización con las características del segmento de mercado debe ser: Producto: elaborado a base de alimentos que contienen principios activos, que permiten proteger, detener y disminuir enfermedades y dolencias. Precio: La estrategia será la de descremado de mercado, ingresar inicialmente con un precio relativamente alto, por las características únicas del producto. Plaza: canal indirecto a través de las bodegas, mercados locales y supermercados de Huancayo y El Tambo. Promoción: Publicidad dirigida a los consumidores, estimulando la demanda por la calidad y la marca específica.

\section{DISCUSIÓN}

De los descriptores utilizados para determinar el segmento de mercado (Nivel educativo, ingreso familiar y estilo de vida), los que pudieron relacionarse con el criterio base: Beneficios buscados en los alimentos con características nutraceúticas, fueron: El nivel educativo los integrantes que tienen un nivel educativo secundario y superior, valoran significativamente el criterio base, en comparación a aquellos que tienen un nivel educativo primario, quienes no tienen interés por el mismo; y el ingreso familiar mensual el segmento está conformado por aquellos que tienen los siguientes ingresos (igual o mayor a S/. 1000,00 pero menos de S/. 2 000,00; igual o mayor S/. 2 000,00 pero menos de S/. 3 000,00 y más de S/. 3 000,00), quienes valoran el criterio base. Obteniéndose un segmento en los dos distritos metropolitanos de $\mathbf{7 4}$ $\mathbf{6 4 5}$ habitantes que representa el $\mathbf{2 3 , 7 0 \%}$ del mercado total, tal como menciona (2), los descriptores permiten realizar segmentaciones a priori, porque definiéndose correctamente el criterio base, se puede seleccionar los descriptores que permiten determinar segmentos de interés estratégico, como es el caso que se identificó un segmento en Ios distritos de Huancayo y El Tambo, interesados y con intención de compra de productos nutraceúticos, como la alcachofa. 


\section{LITERATURA CITADA}

Seguil MCG. 2002. Evaluación de la competitividad en la industrialización y comercialización de la alcachofa en el departamento de Junín. Universidad Nacional Agraria La Molina.

Picón PE, Varela MJ Lévy MJP. 2004. Segmentación de mercados - aspectos estratégicos y metodológicos. Madrid: España.
Hernández SR, Fernández CC, Baptista LP. 1998. Metodología de la investigación. México.

García LF. 1975. Investigación de mercados. España.

Matos MJA. 2005. Plano de calles de la ciudad de Huancayo y distritos aledaños. El Tambo - Perú.

Robles RF. 2000. Alcachofa - nueva alternativa para la agricultura Peruana, Revista Agro enfoque. Lima: Perú. 